\title{
ÍNDICE DE DISCRIMINACIÓN DE LOS ÍTEMS POLITÓMICOS EN LAS PRUEBAS PEDAGÓGICAS
}

\author{
DISCRIMINATION INDEX POLYTOMOUS ITEMS TEACHING IN TESTS
}

Aníbal Cárdenas Ayala ${ }^{16}$, Nadia Limaymanta Álvarez, María Mucha Sedano

Facultad de Educación

\section{RESUMEN}

El presente trabajo de investigación, identificó los niveles de discriminación de los ítems politómicos en las pruebas pedagógicas que se aplican a los alumnos de las instituciones educativas de la Región Junín. Tuvo como objetivos específicos, establecer los factores ponderativos equivalentes a 001 , de los calificativos asignados a los ítems politómicos, elaborar algoritmos de cálculo y determinar el índice de discriminación de los mencionados ítems en las pruebas pedagógicas que se aplican a los alumnos de las instituciones educativas de la Región Junín. Verificando la hipótesis: Existen niveles de discriminación de los ítems politómicos en las pruebas pedagógicas que se aplican a los

alumnos de las instituciones educativas de la Región Junín. La investigación es del tipo tecnológico-aplicada con un diseño descriptivo simple. Se llevó a cabo en la Universidad Nacional del Centro del Perú, Facultad de Educación, a lo largo de un año calendario comprendido entre el 2010-07-01 y el 2011-06-30. Arribó a la conclusión que, son pertinentes el cuadro de equivalencias y el algoritmo de cálculo propuesto, para determinar el poder discriminativo de los ítems politómicos de las pruebas pedagógicas que se aplican a los alumnos de las instituciones educativas de la Región Junín.

Palabras clave: psicometría, edumetría, estadística aplicada a la investigación educativa.

\section{ABSTRACT}

This investigation, identified the levels of discrimination of polytomous items in pedagogic assessments that are applied to students of the educational institutions of Junín region. It had as specific objectives, to establish equivalent factors at 0 or 1 , of scores assigned to polytomous items, to elaborate calculation algorithms and to determine the discrimination index of mentioned items in pedagogic assessment that are applied to students of the educational institutions of Junín region. Verifying the hypothesis: It exists levels of discrimination of polytomous items in pedagogic assessment that are applied to students of the educational institutions of Junin region. The investigation is of technologicalapplied kind with a descriptive simple design. It was made in the Universidad Nacional del Centro del Peru, Education faculty, throughout a year, between 2010-07-01 and 2011-06-30. It arrived to the conclusion that, they are pertinent the equivalences square and the calculation algorithm proposed, to determine discrimination power of polytomous items in pedagogic assessments that are applied to students of the educational institutions of Junín region.

Key words: Psychometric, Edumetric, Statistic applied to the educational investigation

\section{INTRODUCCIÓN}

Entre los años 2004 al 2010, el autor del presente proyecto, realizó investigaciones centradas en

\footnotetext{
16 acardenas@hotmail.com
} 
el cálculo de los estadígrafos psicométricos y edumétricos, tales como, la validez, confiabilidad, dificultad, sensibilidad e índice de discriminación de los diferentes tipos de instrumentos de recolección de datos, habiendo quedado como sugerencias reiterativas, realizar el análisis estadístico y la interpretación del índice de discriminación de los ítems politómicos en las pruebas pedagógicas y fichas de observación.

Por otro lado, el desarrollo de la asignatura de Estadística Aplicada a la Investigación Educativa, en la Unidad de Postgrado de la Facultad de Educación, implica, entre otros temas, el cálculo del estadígrafo edumétrico: índice de discriminación, para ítems dicotómicos; quedando sin desarrollar, por falta de una sistematización matemática, el algoritmo y la interpretación; para los ítems politómicos de las pruebas pedagógicas y fichas de observación.

Entonces, para poder subsanar, en parte, el inconveniente citado líneas arriba, en el presente trabajo de investigación se diseñaron algoritmos de cálculo del índice de discriminación de los ítems politómicos, de las pruebas pedagógicas y fichas de observación que se aplican a los alumnos de las instituciones educativas de la Región Junín.

\section{MATERIAL Y MÉTODOS}

Se tomó como población de la investigación, las diferentes pruebas pedagógicas que aplicaron a sus alumnos, en las diferentes instituciones educativas del nivel secundario de la región Junín, los estudiantes de la Maestría en Enseñanza Estratégica - III Semestre Año Académico 2010; los egresados del pregrado de la Facultad de Educación hasta el año 2010 en sus respectivas tesis; así como a las fichas de observación utilizadas, para evaluaciones procedimentales y actitudinales, por los egresados de la Maestría en Educación, hasta el Año Académico de 2010, las que están consignadas en las tesis de grado.
Para la elección de la muestra se utilizó un diseño muestral no aleatorio dirigido, de tal manera que, la muestra estuvo conformada por una prueba de pensamiento creativo, una prueba pedagógica, ambas politómicas, que se aplicaron a los alumnos de dos instituciones educativas de la UGEL-Huancayo y fichas de observación politómica para la evaluación procedimental en la Universidad Peruana "Los Andes".

Nota: Las pruebas pedagógicas y fichas de observación politómicas fueron bastante escasas.

\section{Tipo de investigación}

Dentro del campo de la investigación educativa y de acuerdo a las características de la hipótesis y los objetivos se enmarcó dentro del tipo de investigación tecnológico-aplicada.

Método básico de la investigación

El método básico fue el descriptivo.

\section{Diseño de la Investigación}

El diseño que se empleó fue el descriptivo, porque resultó imposible el control experimental riguroso de las variables. Además, este diseño se adecuó perfectamente a la presente investigación.

Dentro de los diseños descriptivos se encuentra el diseño descriptivo simple, el que se aplicó para determinar los niveles de discriminación de los ítems politómicos en las pruebas pedagógicas que se aplicaron a los alumnos de las instituciones educativas de la Región Junín.

En el caso concreto de la presente investigación, se aplicaron a las pruebas pedagógicas con ítems politómicos, luego con los datos recolectados para cada ítem, se calcularon los niveles de discriminación, los que fueron analizados e interpretados. Sánchez (2006:103).

El diseño puede diagramarse de la siguiente 
manera:

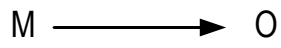

Donde:

M: Pruebas pedagógicas y fichas de observación con ítems politómicos que se aplican a los alumnos de las instituciones educativas de la Región Junín.

0 : Nivel de discriminación de los ítems politómicos de las pruebas pedagógicas y fichas de observación que se aplican a los alumnos de las instituciones educativas de región Junín.

\section{VARIABLES DE LA INVESTIGACIÓN}

Variable independiente: (VI)

Ítems politómicos de las pruebas pedagógicas y fichas de observación que se aplican a los alumnos de las instituciones educativas de Región Junín.

Variable dependiente: (VD)

Niveles de discriminación de los ítems politómicos de las pruebas pedagógicas y fichas de observación que se aplican a los alumnos de las instituciones educativas de región Junín.

\section{TÉCNICAS E INSTRUMENTOS RECOPILACIÓN DE DATOS}

Los instrumentos seleccionados fueron tres: una prueba de pensamiento creativo, una prueba pedagógica, y una ficha de observación para una evaluación procedimental. Los detalles y especificaciones de cada una de ellas son:

Pianto Cencia, Julia y Tinoco Tovar, Liz (egresadas del pregrado); en la I.E. "La Victoria" - El Tambo; en el Área de C.T.A. $4^{\circ}$ grado de secundaria. Durante el III bimestre del Año Académico Escolar 2006. Anexo (1): prueba de pensamiento creativo.

Gamero Tello, Victoria, (egresada de maestría); en la I.E. Mariscal Castilla - El Tambo; en la asignatura de Matemática, Nivel Secundario, Tercer Grado. Durante el II bimestre del Año Académico Escolar 2010. anexo (2): prueba pedagógica.

Tito Ortega, Erwin (egresado de maestría); en la Universidad Peruana "Los Andes"-Facultad de Ciencias de la Salud, E.A.P. de Medicina Humana - Huancayo; en la asignatura de Ginecología y Obstetricia. Durante el $X$ Semestre del Año Académico 2002. anexo (3): ficha de observación-evaluación procedimental.

Se consideraron a estos instrumentos de recolección de datos, debido a que los resultados de los estadígrafos psicométricos calculadoscoeficiente de confiabilidad, coeficiente de validez-fueron óptimos para los objetivos del presente estudio.

\section{PROCEDIMIENTOS DE RECOPILACIÓN DE DATOS}

Los docentes mencionados líneas arriba fueron capacitados (oportunamente) en el diseño, elaboración y aplicación de pruebas. Así como para el análisis estadístico y el cálculo de sus estadígrafos psicométricos de confiabilidad y validez. Los tres instrumentos de recolección de datos, fueron aplicados por los docentes a sus respectivos estudiantes, en sus respectivas instituciones educativas; las docentes, Pianto y Tinoco, hicieron la aplicación como parte de su trabajo de tesis de pregrado; la docente Gamero, hizo la aplicación como parte de su labor pedagógica, es más, como parte de un proceso de entrenamiento propio del desarrollo de la asignatura de Estadística Aplicada a la Investigación Educativa, de la Maestría en 
Educación-Enseñanza Estratégica; finalmente, el docente Tito, hizo lo propio como parte de su trabajo de tesis de maestría en Didáctica Universitaria.

\section{TÉCNICAS $Y$ PROCEDIMIENTOS PROCESAMIENTO Y ANÁLISIS DE DATOS}

Siendo la investigación de carácter cuantitativo, en primer lugar fueron calculados, mediante procedimientos estadísticos, el paquete estadístico SPSS y la aplicación de las fórmulas respectivas, los estadígrafos psicométricos de confiabilidad y validez de los tres instrumentos, porque la condición sine qua non de todo instrumento de recolección de datos, previo al cálculo de otros estadígrafos psicométricos y edumétricos es que sean confiables y válidos. Los resultados, pueden observarse en los Anexos respectivos.

No obstante, a manera de comentario general se tiene que:

Todos los coeficientes de confiabilidad de las pruebas se calcularon aplicando la fórmula alfa de Crombach, siendo los resultados mayores 0 iguales que 0,60 ; por lo que las tres pruebas son confiables.

Todos los coeficientes de validez de criterio (predictiva), de cada uno de los ítems de cada una de las pruebas, fueron calculados aplicando la fórmula de correlación item-total, siendo los resultados mayores o iguales que 0,2000 ; por lo que las tres pruebas son válidas.

\section{RESULTADOS Y DISCUSIÓN}

Análisis psicométrico de las pruebas pedagógicas y ficha de observación

Siendo la investigación de carácter cuantitativo, en primer lugar fueron calculados, mediante procedimientos estadísticos, el paquete estadístico SPSS y la aplicación de las fórmulas respectivas, los estadígrafos psicométricos de confiabilidad y validez de los tres instrumentos tomados como espacio muestral de la presente investigación, porque la condición sine qua non de todo instrumento de recolección de datos, es que sea confiable y válido. Los resultados, pueden observarse en los anexos respectivos. (ver anexos)

Todos los coeficientes de confiabilidad de las pruebas se calcularon aplicando la fórmula alfa de Crombach, siendo todos los resultados, mayores o iguales que 0,60; por lo que las tres pruebas son confiables.

$A C=[k / k-1][1-(s i 2 / s t 2)]$

Donde:

AC: coeficiente de confiabilidad

$\mathrm{k}$ : número de ítems del instrumento.

si2 : sumatoria de varianzas individuales de cada ítem.

st2 : varianza total del instrumento.

Todos los coeficientes de validez de criterio (predictiva), de cada uno de los ítems de cada una de las pruebas, fueron calculados aplicando la fórmula de correlación item-total, siendo todos los resultados, mayores o iguales que 0,2000; por lo que las tres pruebas son válidas.

$r$ (corregido $)=(r$ (crudo) $. s t-s i) /(s t 2+s i 2-2 r$ (crudo).st .si) $1 / 2$

Donde:

r (corregido) : coeficiente de validez del ítem.

$r$ (crudo) : coeficiente de correlación de Pearson.

st : desvío del puntaje total.

si : desvío del ítem.

st2 : varianza del puntaje total. 
si2 : varianza del ítem.

Análisis edumétrico de las pruebas pedagógicas y ficha de observación

Propuesta de algoritmos para el cálculo del poder discriminativo de los ítems politómicos de los instrumentos de recolección de datos

Conceptualmente un ítem dicotómico es aquel cuya calificación es de cero (0) si está mal respondido, y de uno (1) si está bien respondido; en contra posición, un ítem politómico es aquel cuya calificación es de cero (0) si está mal respondido, pero si está bien respondido puede tener calificativos de $1 ; 2 ; 3 ; 4 ; \ldots$ u otros valores enteros o inclusive fraccionarios.

Para calcular el poder discriminativo de los ítem mediante el método del coeficiente de correlación punto serial (rp.bis), implica que todos los ítem de las pruebas sean dicotómicos (0 ó 1) y como el objetivo general del presente trabajo de investigación es identificar los niveles de discriminación de los ítem politómicos en las pruebas, se propuso, en primer lugar, establecer equivalencias entre ítems politómicos y dicotómicos, luego, factores ponderativos equivalentes a 001 , de los calificativos asignados a los ítems politómicos de las pruebas, todo esto se plasma en el siguiente cuadro de equivalencias, para después realizar los cálculos del poder discriminativo de cada uno de los ítems politómicos que conforman cada una de las pruebas tomadas como espacio muestral de la presente investigación.

Cuadro de equivalencias de ítem politómicos con dicotómicos

\begin{tabular}{lcl}
\hline İtem & Calificativo real & $\begin{array}{l}\text { Calificativo } \\
\text { equivalente a } \\
\text { dicotómico } \\
\text { factor ponderativo }\end{array}$ \\
\hline Dicotómico & Mal respondido: 0 & Mal respondido: 0 \\
& Bien respondido:1 & Bien respondido:1 \\
Politómico & Mal respondido: 0 & Mal respondido: 0,0 \\
& Bien respondido:1 & Bien respondido:0,5 \\
Politómico & 2 & 1,0 \\
& Mal respondido: 0 & Mal respondido:0,00 \\
& Bien respondido:1 & Bien respondido:0,34 \\
& 2 & 0,67 \\
Politómico & Mal respondido: 0 & Mal respondido: 0,00 \\
& Bien respondido:1 & Bien respondido:0,25 \\
& 2 & 0,50 \\
3 & 0,75 \\
4 & 1,00 \\
\hline
\end{tabular}

Fuente: Elaboración del autor

El cálculo del poder discriminativo de cada uno de los ítems, se realizó a través del coeficiente de correlación punto serial (rp.bis), cuya fórmula es:

Poder discriminativo $(P D)=r$ p.bis $=(p i-X t) / s t$ .(p/q) $1 / 2$

Donde:

pi : promedio de las puntuaciones totales de los estudiantes que respondieron bien el ítem analizado.

Xt : promedio de las puntuaciones totales de todos los estudiantes considerados en el análisis.

st : desvío de las puntuaciones totales de todos los estudiantes considerados en el análisis.

p y $q$ : proporciones de los estudiantes que, respondieron bien, mal u omitieron el ítem analizado.

Esta fórmula se aplica directamente cuando los ítems son dicotómicos, pero cuando los ítems son politómicos, primero se tienen que establecer las equivalencias de ítem politómico a dicotómico (con el cuadro de equivalencias propuesto), lo que implica que a la matriz de ítem politómicos reales (con valores de 0; 1; 2; 3 ó 4) se la modifique para obtener una nueva matriz de ítems equivalentes a dicotómicos, mediante 
una multiplicación del calificativo real del ítem por el factor ponderativo, lo que da como resultado ítems ponderados, asi como un calificativo TOTAL modificado. A esta matriz de ítems ponderados, cuyos valores, ahora, están comprendidos entre cero (0) y uno (1), tal como se aprecian en los cuadros $0 ; 0,50 ; 1,00$; ó $0 ; 0,25 ; 0,50 ; 0,75$; 1,00 - (ver anexos) hay que agregarle a cada columna del ítem ponderado otra columna que se va a denominar TOTAL ÍTEM (X), la cual es el resultado de la multiplicación de cada rubro de la columna TOTAL por el valor del ítem ponderado; con estas modificaciones, los componentes de la fórmula para el cálculo del poder discriminativo, quedarían como:

Poder discriminativo $(P D)=r p \cdot b i s=(p i-X t) / s t$ .(p/q) $1 / 2$

\section{Donde:}

pi : promedio de las puntuaciones totales de los estudiantes que respondieron

bien el ítem analizado; $p i=$ [Suma TOTAL ÍTEM(X) / Suma ÍTEM(X)]

$X t$ : media de las puntuaciones totales de todos los estudiantes considerados en el análisis; Media del TOTAL modificado.

st : desvío de las puntuaciones totales de todos los estudiantes considerados en el análisis; Desvío del TOTAL modificado.

p y $q$ : proporciones de los estudiantes que, respondieron bien, mal u omitieron el ítem analizado;

$p=$ [Suma ÍTEM $(X) /$ total de participantes $]$

$q=[($ Total de participantes - Suma ÍTEM(X) $)$ / total de participantes]

El valor obtenido también debe ser positivo y mayor o igual a 0,20 para aceptar el poder discriminativo del ítem.

\section{RESULTADOS}

Los coeficientes de poder discriminativo de cada uno de los ítems de los instrumentos de recolección de datos seleccionados como parte de la muestra fueron calculados mediante la aplicación de la fórmula y con la ayuda del paquete informático estadístico (SPSS 15). Ver anexos.

\section{DISCUSIÓN}

Discusión de resultados para la prueba de creatividad

\begin{tabular}{lcl}
\hline Items & $\begin{array}{c}\text { Poder de } \\
\text { discriminación }\end{array}$ & \multicolumn{1}{c}{ Comentario } \\
\hline Ítem 01 & 0.27 & Discriminación intermedia \\
Ítem 02 & 0.36 & Discriminación aceptable \\
Ítem 03 & 0.412 & Discriminación muy buena \\
Ítem 04 & 0.278 & Discriminación intermedia \\
Ítem 05 & 0.218 & Discriminación intermedia \\
Ítem 06 & 0.511 & Discriminación muy buena \\
Ítem 07 & 0.33 & Discriminación aceptable \\
Ítem 08 & 0.30 & Discriminación aceptable \\
Ítem 09 & 0.30 & Discriminación aceptable \\
Ítem 10 & 0.32 & Discriminación aceptable \\
\hline
\end{tabular}

Como se aprecia, los coeficientes de poder de discriminación son positivos y mayores que 0,20 ; de donde se puede inferir que, cada uno de los ítems establecen diferencias entre los estudiantes con niveles altos y bajos en la habilidad y aptitud en el que fueron evaluados.

Por otro lado, los coeficientes de poder discriminativo de la prueba de creatividad, representan el grado de diferenciación, separación, distanciamiento o dispersión de los resultados observados mediante las respuestas que, los estudiantes del $4^{\circ}$ grado de secundaria, dieron a cada pregunta planteada. Las respuestas acertadas, correctas 0 buenas provinieron de aquellos estudiantes que mostraron una creatividad adecuada en el total de la prueba, y que las respuestas erradas u omitidas provinieron del grupo de estudiantes que presentaron una 
deficiente creatividad.

Discusión de resultados para la prueba pedagógica

\begin{tabular}{|c|c|c|}
\hline Ítems & $\begin{array}{c}\text { Poder de } \\
\text { discriminación }\end{array}$ & Comentario \\
\hline Ítem 01 & 0,33 & $\begin{array}{l}\text { Discriminación } \\
\text { aceptable }\end{array}$ \\
\hline Ítem 02 & 0,44 & $\begin{array}{l}\text { Discriminación } \\
\text { muy buena }\end{array}$ \\
\hline Ítem 03 & 0,52 & $\begin{array}{l}\text { Discriminación } \\
\text { muy buena }\end{array}$ \\
\hline Ítem 04 & 0,82 & $\begin{array}{l}\text { Discriminación } \\
\text { muy buena }\end{array}$ \\
\hline Ítem 05 & 0,58 & $\begin{array}{l}\text { Discriminación } \\
\text { muy buena }\end{array}$ \\
\hline Ítem 06 & 0,60 & $\begin{array}{l}\text { Discriminación } \\
\text { muy buena }\end{array}$ \\
\hline Ítem 07 & 0,46 & $\begin{array}{l}\text { Discriminación } \\
\text { muy buena }\end{array}$ \\
\hline Ítem 08 & 0,77 & $\begin{array}{l}\text { Discriminación } \\
\text { muy buena }\end{array}$ \\
\hline Ítem 09 & 0,51 & $\begin{array}{l}\text { Discriminación } \\
\text { muy buena }\end{array}$ \\
\hline Ítem 10 & 0,50 & $\begin{array}{c}\text { Discriminación } \\
\text { muy buena }\end{array}$ \\
\hline
\end{tabular}

Como se puede apreciar los coeficientes de poder de discriminación son positivos y mayores que 0,20 ; de donde se puede inferir que, cada uno de los ítems establecen diferencias entre los estudiantes con niveles altos y bajos en habilidades, aptitudes y conocimientos en matemática.

Por otro lado, los coeficientes de poder discriminativo de la prueba pedagógica, representan el grado de diferenciación, separación, distanciamiento 0 dispersión de los resultados observados mediante las respuestas que los estudiantes del $3^{\circ}$ grado de secundaria dieron a cada pregunta planteada. Las respuestas acertadas, correctas o buenas provinieron de aquellos estudiantes que mostraron un rendimiento adecuado en el total de la prueba, y que las respuestas erradas u omitidas provinieron del grupo de estudiantes que presentaron un rendimiento deficiente en la prueba.

Discusión de resultados para la fichas de observación-
Evaluación procedimental

\begin{tabular}{ccc}
\hline Ítems & $\begin{array}{c}\text { Poder de } \\
\text { discriminación }\end{array}$ & \multicolumn{1}{c}{ Comentario } \\
\hline Item 01 & 0,56 & Discriminación muy buena \\
Ítem 02 & 0,66 & Discriminación muy buena \\
Ítem 03 & 0,59 & Discriminación muy buena \\
Ítem 04 & 0,26 & Discriminación intermedia \\
Ítem 05 & 0,28 & Discriminación intermedia \\
Ítem 06 & 0,40 & Discriminación muy buena \\
Ítem 07 & 0,26 & Discriminación intermedia \\
Ítem 08 & 0,28 & Discriminación intermedia \\
Ítem 09 & 0,31 & Discriminación aceptable \\
Ítem 10 & 0,38 & Discriminación aceptable \\
Ítem 11 & 0,45 & Discriminación muy buena \\
Ítem 12 & 0,26 & Discriminación intermedia \\
Ítem 13 & 0,21 & Discriminación intermedia \\
Ítem 14 & 0,24 & Discriminación intermedia \\
Ítem 15 & 0,27 & Discriminación intermedia \\
Ítem 16 & 0,27 & Discriminación intermedia \\
Ítem 17 & 0,27 & Discriminación intermedia \\
Ítem 18 & 0,21 & Discriminación intermedia \\
Ítem 19 & 0,28 & Discriminación intermedia \\
Ítem 20 & 0,32 & Discriminación aceptable \\
\hline
\end{tabular}

Como se puede apreciar los coeficientes de poder de discriminación son positivos y mayores o iguales que 0,20; de donde se puede inferir que, cada uno de los ítems establecieron diferencias entre los estudiantes con niveles altos y bajos en una habilidad, aptitud o conocimiento en el que fueron evaluados.

Por otro lado, los coeficientes de poder discriminativo de la ficha de observación para una evaluación procedimental de estudiantes de medicina humana, representan el grado de diferenciación, separación, distanciamiento 0 dispersión de los resultados observados mediante las respuestas que los estudiantes dieron a cada ítem de observación. Las observaciones acertadas, correctas o buenas provinieron de aquellos estudiantes que también mostraron un rendimiento o desempeño adecuado en toda la observación, y los ítems de observación errados provinieron del grupo de estudiantes deficientes en la observación.

Se elaboró un cuadro de equivalencias de ítems politómicos con ítems dicotómicos, mediante 
la aplicación de factores ponderativos, de las pruebas pedagógicas que se aplican a los alumnos de las instituciones educativas de la Región Junín.

Se diseñaron algoritmos para el cálculo del poder de discriminación de ítem politómicos. de las pruebas pedagógicas que se aplican a los alumnos de las instituciones educativas de la Región Junín.

Se determinó el poder discriminativo de ítems politómicos de las pruebas pedagógicas que se aplican a los alumnos de las instituciones educativas de la Región Junín.

De acuerdo a los análisis y discusión de los resultados, son pertinentes, el cuadro de equivalencias y el algoritmo de cálculo propuesto, para determinar el poder discriminativo de los ítems politómicos de las pruebas pedagógicas que se aplican a los alumnos de las instituciones educativas de la Región Junín.

\section{REFERENCIAS BIBLIOGRÁFICAS}

Ary, Jacobs, Razavieh. Introducción a la investigación pedagógica, Editorial McGraw-Hill, México. D.F.-México. 1992.

Cárdenas Ayala, Aníbal. Pertinencia de los Instrumentos de recolección de datos con los métodos estadísticos de confiabilidad, I.I. FPH-UNCP, trabajo de investigación inédito. Huancayo-Perú. 2007.

I.I.FPH-UNCP, trabajo de investigación inédito. Análisis estadístico de los instrumentos de recolección de datos a través de los estadígrafos de deformación y apuntamiento, Huancayo-Perú. 2008.

I.I.FPH-UNCP, trabajo de investigación inédito. Poder discriminativo, nivel de exigencia y capacidad predictiva de los exámenes de Selección, Huancayo-Perú. 2009.
I.I. FE-UNCP, trabajo de investigación inédito. Relación entre la validez, el poder discriminativo y nivel de dificultad de las pruebas pedagógicas, Huancayo. 2010.

Delgado, Escurra, Torres. La medición en psicología y educación, s/e, Lima-Perú. 2006.

Downie, Heath. Métodos estadísticos aplicados, Editorial Harla, México D.F.-México. 1989.

Gonzalvo, Gonzalo. Diccionario de metodología estadística, Ediciones Morata S.A., Madrid. España. 1989.

Hernández, Fernández, Baptista. Metodología de la investigación, Editorial McGraw-Hill, México. 2003.

Kerlinger, F. Investigación del comportamiento, Editorial Interamericana, México. 1995.

Nunnally, Bernstein. Teoría psicométrica, Editorial Mc Graw-Hill, México. 1995.

Pagano, Robert. Estadística para las ciencias del comportamiento, International Thomson. Editores, México D.F.- México. 1999.

Sánchez Carlesi, Reyes Meza. Metodología y Diseños en la Investigación Científica, Editorial Educativa del INIDE, Lima -Perú. 1996.

Schiefel Bein, Ernesto. Teoría, técnicas, procesos y casos en el planeamiento de la educación. Editorial Ateneo, Buenos Aires- Argentina. 1995.

Tavella, Nicolás. Análisis de los ítems en la construcción de instrumentos psicométricos, Editorial Trillas, México. 1992.

Visauta, Bienvenido, Martori i Cañas, Joan. Análisis estadístico con SPSS para windows, Volumen II, Editorial McGraw-Hill. MadridEspaña. 2004.

Quantitative data processing: Churchman / Ackoff / Arnoff

http://www.yale university.research center.org. usa Septiembre, 2007 